\title{
Impact of Extranodal Extension on Risk Stratification in Papillary Thyroid Carcinoma
}

\author{
Hye In Kim, ${ }^{1, *}$ Jiyeon Hyeon, ${ }^{2, *}$ So Young Park, ${ }^{3}$ Hyeon Seon Ahn, ${ }^{4}$ Kyunga Kim, ${ }^{4}$ Ji Min Han, \\ Ji Cheol Bae, Jung Hee Shin, Jee Soo Kim, ${ }^{6}$ Sun Wook Kim, ${ }^{3}$ Jae Hoon Chung, ${ }^{3}$ \\ Tae Hyuk Kim, ${ }^{3}$ and Young Lyun $\mathrm{Oh}^{7}$
}

Background: The current American Thyroid Association risk-stratification system for papillary thyroid carcinoma (PTC) incorporates the number and size of positive lymph nodes (LNs) but places less weight on extranodal extension (ENE). This study investigated how to incorporate ENE into the current system to predict recurrence better in PTC N1 patients.

Methods: A total of 369 N1 PTC patients without distant metastasis were enrolled. The combination of number of positive LNs and LNs with ENE that had the highest C-index were identified with multivariable Cox proportional hazards models. ENE number was incorporated into the current system considering the recurrence rate and unadjusted and adjusted hazard ratios (HRs) of the subgroups. Kaplan-Meier curves for recurrence based on current and alternative systems were compared by log-rank test.

Results: The recurrence rate for the subgroup with five or fewer positive LNs and one to three ENEs (7/61; $11.5 \%)$ was higher than that of the subgroup with five or fewer positive LNs without ENE $(5 / 129 ; 3.9 \%$; adjusted $\mathrm{HR}=3.42$ [confidence interval (CI) 0.99-11.75]; $p=0.050$ ). In contrast, adjusted HRs of the subgroup with more than five positive LNs and one to three ENEs (2.33 [CI 0.52-10.35]) or with four or more ENEs (3.86 [CI 1.0514.17]) were not higher than those of the subgroup with more than five LNs without ENE (4.47 [1.16-17.19]). Incorporating ENE into the current system as an intermediate-risk group yielded a lower log-rank $p$-value $(0.05$ vs. $0.01)$ than the current system.

Conclusions: The presence of ENE in low volume LN metastasis confers an intermediate risk of recurrence. Incorporating ENE into the current system allows more accurate decisions regarding further management of PTC N1 patients.

Keywords: papillary thyroid carcinoma, recurrence, extranodal extension, risk-stratification system

\section{Introduction}

$\mathbf{P}$ HYSICIANS CURRENTLY USE the risk-stratification system of the American Thyroid Association (ATA) to determine further management options for patients with papillary thyroid carcinoma (PTC), including radioactive iodine (RAI) treatment, degree of thyrotropin (TSH) suppression, and surveil- lance. The currently used system incorporates two lymph node (LN) criteria to stratify nodal disease: the number and largest size of positive LNs (1).

The presence of extranodal extension (ENE), which is a well-known adverse prognostic factor for various cancer types (2-4), has emerged as an important risk factor for the recurrence of PTC (5-8). Furthermore, Leboulleux et al.

\footnotetext{
${ }^{1}$ Division of Endocrinology and Metabolism, Department of Medicine, Samsung Changwon Hospital, Sungkyunkwan University School of Medicine, Changwon, Republic of Korea.

${ }^{2}$ Department of Pathology, Incheon St Mary's Hospital, College of Medicine, The Catholic University of Korea, Seoul, Republic of Korea.

${ }^{3}$ Division of Endocrinology and Metabolism, Department of Medicine, Thyroid Center; ${ }^{4}$ Statistics and Data Center, Research Institute for Future Medicine; ${ }^{5}$ Department of Radiology; ${ }^{6}$ Division of Breast and Endocrine Surgery, Department of Surgery; Department of Pathology; Samsung Medical Center, Sungkyunkwan University School of Medicine, Seoul, Republic of Korea.

*These authors contributed equally to this work.

(C) Hye In Kim et al. 2019; Published by Mary Ann Liebert, Inc. This Open Access article is distributed under the terms of the Creative Commons License (http://creativecommons.org/licenses/by/4.0), which permits unrestricted use, distribution, and reproduction in any medium, provided the original work is properly cited
} 
suggested that the number of ENEs is associated with an increased risk of recurrence of PTC (9). Based on this study, the ATA guidelines state that patients with LNs with three or fewer ENEs are at low risk of recurrence (2\%), while those with LNs with more than three ENEs are at high risk of recurrence (about 40\%) (1).

However, ENE was not incorporated in the current ATA risk-stratification system for recurrence for the following reasons. First, the presence and number of ENEs are closely linked to the number of positive LNs, which is already included as a variable in the system $(1,10)$. In addition, the lack of definition of an ENE, the high average number of LNs with ENE, and no evaluation of largest LN size in a previous study (9) made it difficult to incorporate ENEs into the current guidelines. Considering the prognostic significance of ENE, an alternative ATA riskstratification system that includes ENE criteria is needed. However, most studies have focused only on the fact that ENE is a risk factor of recurrence, and have not investigated how to incorporate ENE into the current ATA risk-stratification system taking multi-collinearity into consideration.

Accordingly, this study evaluated the impact of the presence and number of ENEs on recurrence in patients with PTC, with an emphasis on how to incorporate this information into the current ATA risk-stratification system.

\section{Methods}

\section{Study subjects}

Records of 958 patients with PTC who underwent total thyroidectomy and RAI treatment between April 1, 2012, and December 31, 2014, at the Samsung Medical Center were reviewed retrospectively. Exclusion criteria included age $<18$ years at time of surgery $(n=5)$, N0 disease $(n=113)$, tumor size $\leq 1.0 \mathrm{~cm}(n=366)$, and lack of available data for LNs $(n=105)$. A total of 369 patients were enrolled in the present study (Supplementary Fig. S1). This study was approved by the Institutional Review Board of the Samsung Medical Center (IRB no. 2018-02-034).

\section{Evaluation of $L N s$}

All enrolled patients underwent central compartment node dissection with or without lateral compartment node dissection. According to the surgeon's judgment based on the ATA guidelines, both prophylactic central neck dissection (CND) and selective CND were performed $(1,11)$. In the case of prophylactic CND, usually an ipsilateral paratracheal dissection was performed. A bilateral paratracheal dissection was performed only if there was documented contralateral paratracheal LN metastasis or N1b disease. "Berry picking" resection, in which just grossly positive LNs are excised (12), was not performed in any of the enrolled patients. All harvested LNs were fixed in $10 \%$ phosphate-buffered formalin, embedded in paraffin, and split evenly. Pathologic slides stained with hematoxylin and eosin were reviewed by two experienced pathologists. In addition to size and total number of positive LNs, the presence and number of ENEs were also evaluated. ENE was defined as the extension of metastatic cells beyond the nodal capsule into the perinodal soft tissue, which is the definition used in several earlier studies (Fig. 1) $(2,3,6)$. The extent or diameter of individual ENEs was not measured.

\section{Study design and statistical analysis}

The outcome of the current study was structural persistent/ recurrent disease, which was defined as cytopathology-proven disease or biochemically incomplete evidence (basal serum thyroglobulin $>1.0 \mathrm{ng} / \mathrm{mL}$ ) with a lesion highly suspicious of being recurrent on two serial imaging studies (thyroid ultrasonography, neck computed tomography [CT], whole-body RAI scan, or positron emission tomography-CT). This definition thus referred to both recurrent and biochemically persistent disease that was newly identified in imaging studies. Recurrence-free survival was defined as the time interval (in months) between the initial surgery date and the most recent follow-up date for patients without structural persistent/recurrent disease (13-17).

To resolve the multi-collinearity problem, possible combinations of positive LN number and ENE number were derived. For this, two fixed categories ( $\leq 5$ positive LNs and $>5$ positive $\mathrm{LNs}$ ) for LN number and three non-fixed categories (no ENE, $1-K$ ENEs, $\geq K+1$ ENEs) for ENE number were serially matched. $K$ is an arbitrary natural number ranging from 2 to 10 , and it is used to specify non-fixed categories of ENEs. A total of nine combinations each composed of six subgroups were derived (Table 2).

Cox proportional hazards models were constructed to assess the association between the various combinations and recurrence, with or without adjusting for conventional prognostic factors such as age, sex, gross extrathyroidal extension (ETE), and therapeutic RAI (defined as a RAI dosage of $\geq 100$ $\mathrm{mCi}$ ) (1). Then, the $\mathrm{C}$-index of the Cox proportional hazards models was compared to identify the best combination for predicting recurrence. The $\mathrm{C}$-index is a statistical method quantifying the goodness of models with binary outcome, which is equal to the area under the receiver operating characteristic (ROC) curve. The combination associated with the highest $\mathrm{C}$-index in the multivariable Cox proportional hazard models was selected (18-20). Last, by comparing recurrence rate and unadjusted and adjusted hazard ratios (HRs) for the risk of structural persistent/recurrent disease of subgroups, the effect of ENE when incorporated into the current ATA risk-stratification system was estimated. The Kaplan-Meier curve for recurrence derived from the proposed system was compared with that of the current system by the log-rank test.

All categorical variables are presented as numbers and percentages, while continuous variables are presented as means \pm standard deviations $(S D s)$ if the variable followed a normal distribution, and medians with interquartile range (IQR) for continuous variables that did not follow a normal distribution. All statistical analyses were performed using SAS v9.4 (SAS Institute, Cary, NC). The level of significance was set at $p<0.05$ for two-sided tests.

\section{Results \\ Baseline characteristics}

Baseline characteristics of the 369 patients are presented in Table 1. The median age was 42.0 years (IQR 33.5-53.0 

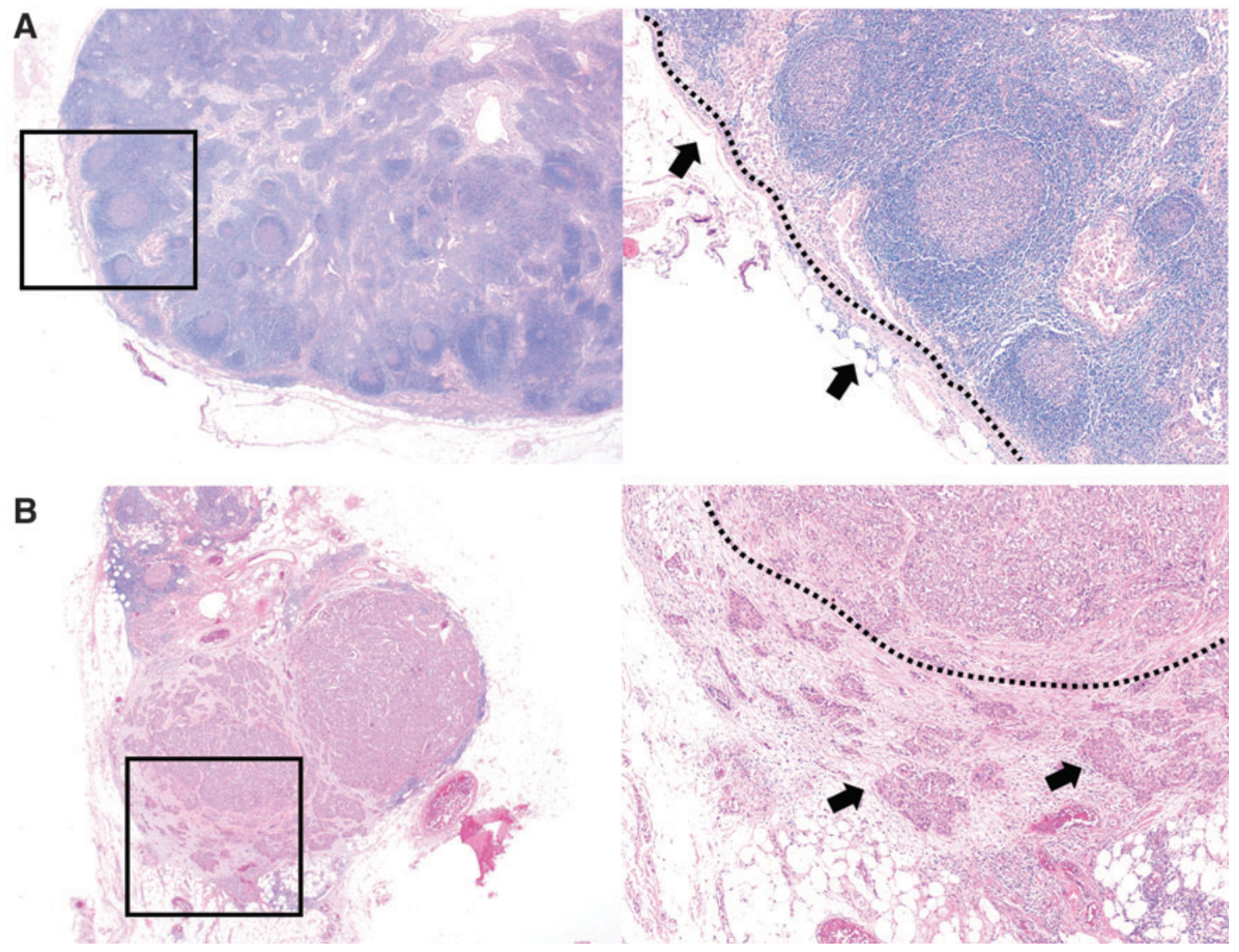

FIG. 1. Example of lymph nodes (LNs) negative (A) or positive (B) for extranodal extension (ENE) in patients with papillary thyroid cancer (PTC; hematoxylin and eosin stain; original magnification $\times 10$ and $\times 40$ ). (A) ENE negative: no tumor cell invading the perinodal soft tissue (arrows) and an intact nodal capsule (dotted line). (B) ENE positive: tumor cells invading the perinodal soft tissue (arrows) beyond the nodal capsule (dotted line).

years), and $63 \%(n=234)$ of patients were female. The median largest positive $\mathrm{LN}$ size was $0.6 \mathrm{~cm}$ (IQR $0.3-1.1 \mathrm{~cm}$ ), and the median number of positive LNs was 5 (IQR 2-10), respectively. ENE was detected in $193(52.3 \%)$ of the 369 patients, and the median number of ENEs was 3.0 (IQR 1.0-6.0).

\section{Table 1. Patient Characteristics}

\begin{tabular}{lc}
\hline Characteristic & \\
\hline Female, $n(\%)$ & $234(63 \%)$ \\
Age (years), median (IQR) & $42.0(33.5-53.0)$ \\
ETE, $n(\%)$ & \\
$\quad$ No ETE & $67(18 \%)$ \\
Minimal or T3b & $256(69 \%)$ \\
T4 & $46(12 \%)$ \\
Tumor size (cm), median (IQR) & $1.6(1.3-2.3)$ \\
Aggressive histology, $n(\%)$ & $14(4 \%)$ \\
Total positive LNs, median (IQR) & $5(2-10)$ \\
Patients with >5 positive LNs, $n(\%)$ & $166(48 \%)$ \\
Largest LN size (cm), median (IQR) & $0.6(0.3-1.1)$ \\
Presence of ENE, $n(\%)$ & $193(52.3 \%)$ \\
Therapeutic RAI, $n(\%)$ & $238(64.5 \%)$ \\
Follow-up length (months), median (IQR) & $40(32-48)$ \\
\hline
\end{tabular}

IQR, interquartile range; ETE, extrathyroidal extension; LN, lymph node; ENE, extranodal extension; RAI, radioactive iodine.

\section{Combination of positive $L N$ number and ENE number}

As expected, the numbers of positive LNs and ENEs were closely related (Pearson's correlation coefficient $=0.66$, $p<0.001$; data not shown). Among nine multivariable Cox proportional hazards models with various combinations of the number of positive LNs and ENEs, combination $2(\leq 5$ LNs without ENEs; $\leq 5 \mathrm{LNs}$ and $1-3$ ENEs; $\leq 5 \mathrm{LNs}$ and $\geq 4$ ENEs; $>5$ LNs without ENEs; $>5$ LNs and 1-3 ENEs; >5 LNs and $\geq 4$ ENEs) had the highest C-index (0.719 \pm 0.049 ; Table 2).

\section{Impacts of ENE on structural persistent/ recurrent disease}

During the median follow-up period of 40 months, structural persistent/recurrent disease was observed in $35(9.49 \%)$ patients. The structural persistent/recurrent disease occurred in local $\mathrm{LN}(n=29 ; 6$ cases in central $\mathrm{LN}$ and 23 cases in lateral LN), local soft tissue except $\mathrm{LN}(n=5)$, and a distant area ( $n=1$, the lung) (21). The recurrence rate for the subgroup with five or fewer LNs and one to three ENEs (7/61; $11.5 \%$ ) was higher than that for the subgroup with five or fewer LNs without ENE (5/129; 3.9\%; unadjusted HR=3.19 [confidence interval (CI) 1.01-10.06], $p=0.047$; adjusted $\mathrm{HR}=3.42$ [CI 0.99-11.75], $p=0.050$ ) and similar to that of the subgroup with more than five LNs without ENE 
Table 2. Possible Combinations of Numbers of Positive Lymph Nodes and Numbers OF LYMPH NODES WITH ENE AND THE C-INDEX

\begin{tabular}{|c|c|c|c|c|c|c|c|}
\hline \multirow[b]{3}{*}{ Combination $K^{\mathrm{a}}$} & Subgroup 1 & Subgroup 2 & Subgroup 3 & Subgroup 4 & Subgroup 5 & Subgroup 6 & \multirow[b]{3}{*}{$C$-index ${ }^{\mathrm{b}} \pm S E$} \\
\hline & \multicolumn{3}{|c|}{ Positive LNs $\leq 5$} & \multicolumn{3}{|c|}{ Positive LNs $>5$} & \\
\hline & \multicolumn{3}{|c|}{ Number of ENEs } & \multicolumn{3}{|c|}{ Number of ENEs } & \\
\hline Combination 1 & 0 & $1-2$ & $\geq 3$ & 0 & $1-2$ & $\geq 3$ & $0.711 \pm 0.049$ \\
\hline Combination 2 & $\mathbf{0}$ & 1-3 & $\geq 4$ & $\mathbf{0}$ & 1-3 & $\geq 4$ & $0.719 \pm 0.049$ \\
\hline Combination 3 & 0 & $1-4$ & $\geq 5$ & 0 & $1-4$ & $\geq 5$ & $0.710 \pm 0.049$ \\
\hline Combination 4 & 0 & $1-5$ & $\geq 6$ & 0 & $1-5$ & $\geq 6$ & $0.709 \pm 0.049$ \\
\hline Combination 5 & 0 & $1-6$ & $\geq 7$ & 0 & $1-6$ & $\geq 7$ & $0.709 \pm 0.049$ \\
\hline Combination 6 & 0 & $1-7$ & $\geq 8$ & 0 & $1-7$ & $\geq 8$ & $0.713 \pm 0.049$ \\
\hline Combination 7 & 0 & $1-8$ & $\geq 9$ & 0 & $1-8$ & $\geq 9$ & $0.716 \pm 0.049$ \\
\hline Combination 8 & 0 & $1-9$ & $\geq 10$ & 0 & $1-9$ & $\geq 10$ & $0.709 \pm 0.049$ \\
\hline Combination 9 & 0 & $1-10$ & $\geq 11$ & 0 & $1-10$ & $\geq 11$ & $0.717 \pm 0.049$ \\
\hline
\end{tabular}

Combination 2 indicates the combination with the highest $\mathrm{C}$-index (shown in bold).

${ }^{\mathrm{a}}$ Combination $\mathrm{K}$ was derived by matching three categories $(0,1-K, \geq K+1$, where $K$ ranged from 2 to 10$)$ for ENE number and two fixed categories ( $\leq 5$ positive LNs, $>5$ positive LNs) for the number of positive LNs.

${ }^{\mathrm{b}} \mathrm{C}$-index of multivariable Cox proportional hazard model with the combination included as the main variable for recurrence.

$S E$, standard error.

(recurrence rate $6 / 47 ; 12.8 \%$; unadjusted $\mathrm{HR}=3.64[\mathrm{CI}$ $1.11-11.95$ ], $p=0.033$; adjusted HR $=4.47$ [CI 1.16-17.19], $p=0.028)$. The latter subgroup is classified as an intermediaterisk group in the current risk-stratification system. Adequate statistical analysis was not available for the subgroup with five or fewer LNs and four or more ENEs because of the small size of this subgroup and lack of outcome data $(0 / 2)$.

Recurrence rates of the three subgroups with more than five LNs (intermediate risk in the current system) were $12.7 \%$ (6/47), 8.1\% (4/49), and 14.1\% (11/78), respectively. These values are higher than those obtained for the subgroup with five or fewer LNs without ENE. However, unadjusted and adjusted HRs of the subgroup with more than five LNs with one to three ENEs (2.18 [CI 0.58-8.14] and 2.33 [CI 0.52$10.35]$ ) or with four or more ENEs (4.46 [CI 1.59-12.54] and 3.86 [CI 1.05-14.17]) were not higher than those of the subgroup with more than five LNs without ENEs (3.64 [CI 1.11-11.95] and 4.47 [CI 1.16-17.19]; Table 3).

\section{Incorporating ENE into the ATA risk-stratification system}

The process of incorporating ENE into the current riskstratification system considering the recurrence rate and

Table 3. Univariable and Multivariate Cox Proportional Hazard Models to Predict Structural Persistent/Recurrent Disease in PTC N1 Patients

\begin{tabular}{|c|c|c|c|c|}
\hline \multirow[b]{2}{*}{ Variable } & \multicolumn{2}{|c|}{ Univariable } & \multicolumn{2}{|c|}{ Multivariable } \\
\hline & $H R[C I]$ & $\mathrm{p}$-Value & $H R[C I]$ & $\mathrm{p}$-Value \\
\hline Age $\geq 55$ years & $1.18[0.53-2.60]$ & 0.673 & $1.07[0.45-2.53]$ & 0.877 \\
\hline Female & $0.60[0.31-1.17]$ & 0.139 & $0.74[0.36-1.52]$ & 0.414 \\
\hline Tumor size (cm) & $1.51[1.15-1.99]$ & 0.003 & $1.37[1.00-1.87]$ & 0.049 \\
\hline \multicolumn{5}{|l|}{ ETE } \\
\hline No ETE & Ref. & & Ref. & \\
\hline Microscopic ETE & $2.46[0.74-8.12]$ & 0.138 & $2.25[0.67-7.55]$ & 0.186 \\
\hline Gross ETE & $2.52[0.60-10.57]$ & 0.204 & $1.88[0.42-8.33]$ & 0.404 \\
\hline Aggressive histology & $0.77[0.10-5.64]$ & 0.798 & $0.57[0.07-4.74]$ & 0.610 \\
\hline Therapeutic RAI & $1.34[0.64-2.80]$ & 0.426 & $1.66[0.77-3.59]$ & 0.193 \\
\hline N1b disease & $1.62[0.83-3.15]$ & 0.154 & $1.00[0.45-2.24]$ & 0.990 \\
\hline \multicolumn{5}{|l|}{ Largest LN size $(\mathrm{cm})$} \\
\hline$<0.2 \mathrm{~cm}$ & Ref. & & Ref. & \\
\hline $0.2-3.0 \mathrm{~cm}$ & $1.55[0.37-6.51]$ & 0.544 & $0.62[0.12-3.17]$ & 0.569 \\
\hline$\geq 3.0 \mathrm{~cm}$ & $21.99[3.06-157.76]$ & 0.002 & $2.45[0.22-26.87]$ & 0.461 \\
\hline \multicolumn{5}{|l|}{ Combinations } \\
\hline$\leq 5 \mathrm{LNs}$, no ENE & Ref. & & Ref. & \\
\hline$\leq 5 \mathrm{LNs}, 1-3 \mathrm{ENEs}$ & 3.19 [1.01-10.06] & 0.047 & $3.42[0.99-11.75]$ & 0.050 \\
\hline$\leq 5 \mathrm{LNs}, \geq 4$ ENEs & - & 0.983 & - & 0.989 \\
\hline$>5$ LNs, no ENE & 3.64 [1.11-11.95] & 0.033 & $4.47[1.16-17.19]$ & 0.028 \\
\hline$>5$ LNs, 1-3 ENEs & $2.18[0.58-8.14]$ & 0.244 & $2.33[0.52-10.35]$ & 0.265 \\
\hline$>5 \mathrm{LNs}, \geq 4 \mathrm{ENEs}$ & $4.46[1.59-12.54]$ & 0.004 & $3.86[1.05-14.17]$ & 0.041 \\
\hline
\end{tabular}

HR, hazard ratio; CI, confidence interval. 


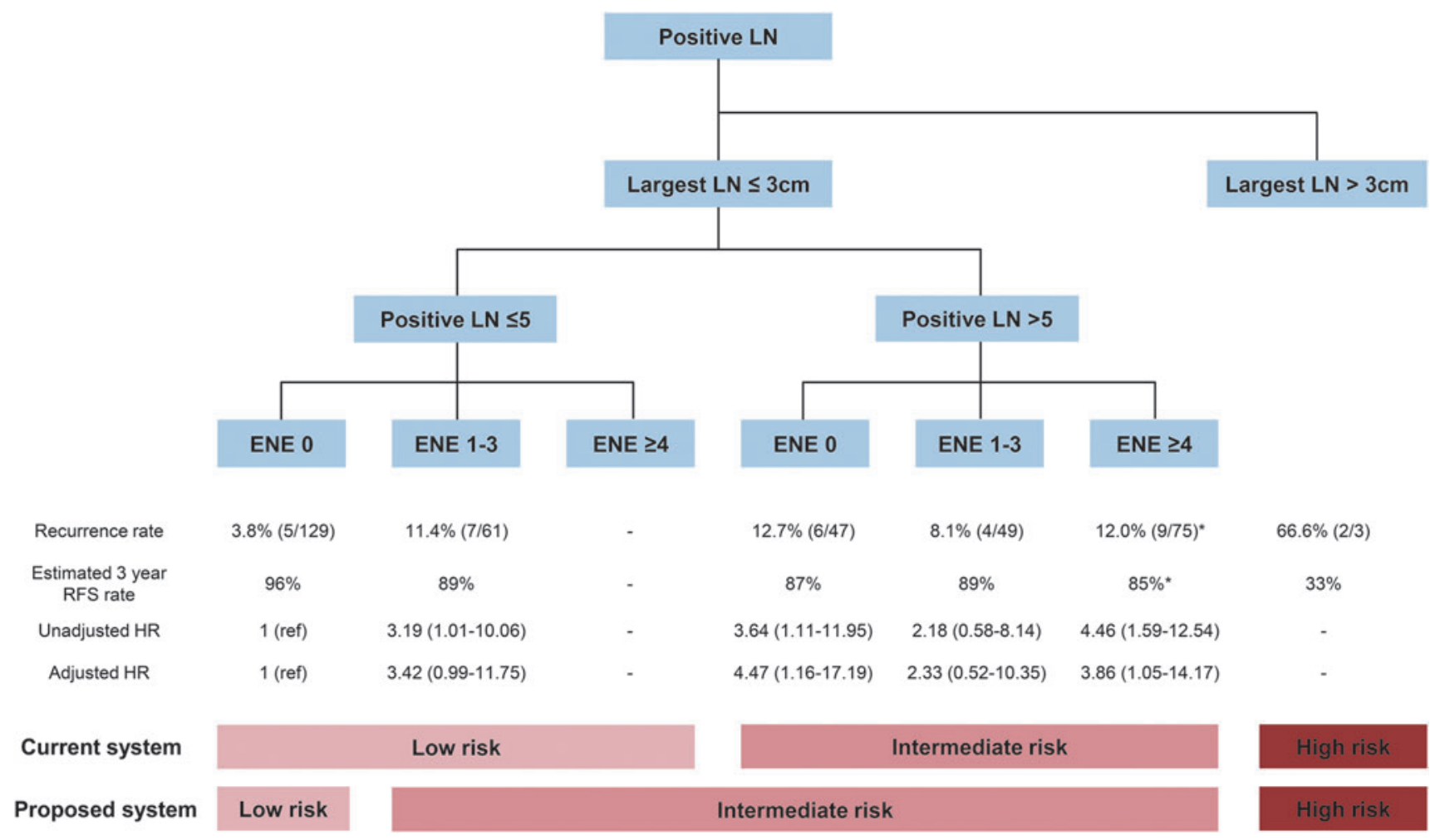

FIG. 2. The process of incorporating ENE number into the current American Thyroid Association (ATA) riskstratification system. *Recurrence rate and estimated three-year RFS rate were calculated after excluding the three patients with the largest LNs (LNs $>3 \mathrm{~cm}$ ) HR, hazard ratio; RFS, recurrence-free survival.

unadjusted and adjusted HRs of each subgroup is illustrated in Figure 2. Given the similar recurrence rate and unadjusted or adjusted HR, a considerable portion of patients with five or fewer LNs $(63 / 192 ; 32.8 \%)$ but with ENE were up-staged from the low- to intermediate-risk group. Patients who had more than five LNs with ENE remained in the intermediaterisk group because there was no further increase in risk of recurrence according to the presence or number of ENEs. The alternative risk-stratification system showed a significant log- rank $p$-value $(p=0.010)$ for the Kaplan-Meier curve for recurrence, while the current ATA risk-stratification system did not $(p=0.050$; Fig. 3).

\section{Discussion}

This study defined the optimal combination of number of ENEs and number of positive LNs composed of six subgroups ( $\leq 5 \mathrm{LNs}$ without ENE; $\leq 5 \mathrm{LNs}$ with $1-3 \mathrm{ENEs} ; \leq 5$

B

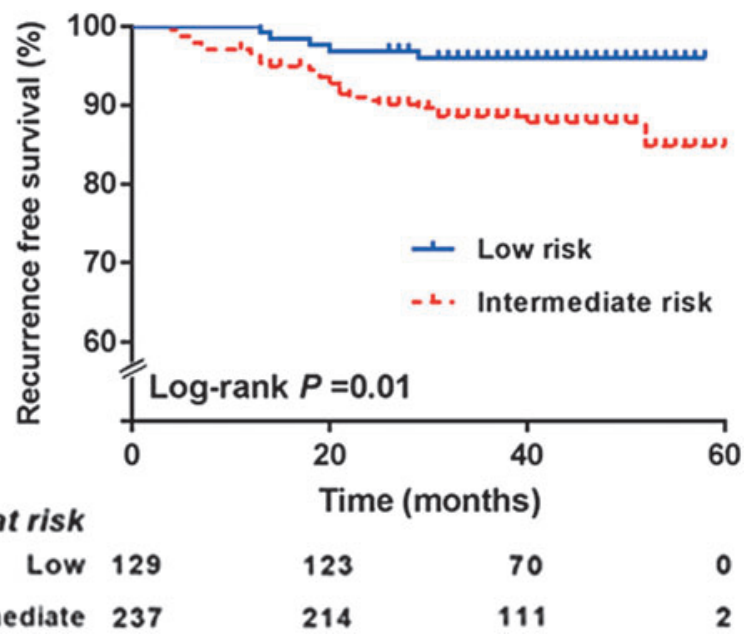

$\begin{array}{rrr}3 & \text { No.at risk } \\ 2 & \text { Low } & 129 \\ 0 & \text { Intermediate } & 237\end{array}$

Intermediate 174

\begin{tabular}{rrrrr} 
No.at risk & \multicolumn{4}{c}{ Time (months) } \\
Low 192 & 178 & 100 & 2 \\
Intermediate & 174 & 159 & 81 & 0
\end{tabular}

60

FIG. 3. Kaplan-Meier curves for structural persistent/recurrent disease in PTC N1 patients according to (A) the current ATA risk stratification system and (B) the alternative system. 
LNs with $\geq 4$ ENEs; $>5$ LNs without ENE; $>5$ LNs with 1-3 ENEs; $>5$ LNs with $\geq 4$ ENEs) to predict the risk of recurrent/ persistent disease better. The subgroup with five or fewer LNs and one to three ENEs had a higher risk of recurrence than the subgroup with five or fewer LNs without ENE, and the risk of recurrence in the subgroup with five or fewer LNs and one to three ENEs was similar to that of the three subgroups with more than five LNs. The presence of ENEs therefore translates to intermediate risk within the current risk-stratification system.

When considering previous studies (5-7,9,22-25), including a recent meta-analysis (8), it seems clear that the presence of ENE is a significant adverse prognostic factor in thyroid cancer. However, the presence and number of ENEs are closely linked with the number of positive LNs, which makes it difficult to incorporate ENEs into the current riskstratification system. Recent ATA guidelines therefore commented that PTC patients with more than three ENEs have a high risk of recurrence, but this is not reflected in the risk-stratification system (1). In contrast to prior studies, this study combined ENE number and positive LN number using the C-index of multivariable Cox proportional hazards models, which made it possible to evaluate ENE risk within the current risk-stratification system. It was found that stratifying the number of ENEs as $0,1-3$, or $\geq 4$ was most appropriate for predicting structural persistent/recurrent disease in the current risk-stratification system.

Among patients with five or fewer positive LNs (low-risk group in the current system), the presence of ENE had an impact on the risk for structural persistent/recurrent disease of PTC, and HRs were similar to those of subgroups with more than five LNs. Furthermore, despite the presence of five or fewer LNs, the recurrence rate in the subgroup with ENE was $>10 \%$, similar to findings for many intermediate groups based on ATA guidelines (minor ETE, 8\%; intrathyroidal PTC $<4 \mathrm{~cm}$ with $B R A F$ mutation, $10 \%$; pathologic positive $\mathrm{LN}$ number $>5,20 \%$ ) (1). This result is in accordance with other studies that reported recurrence rates of $14.5 \%$ (22), $15 \%$ (26), and $22.8 \%$ (27) in PTC patients with ENE. In contrast, Leboulleux et al. reported a recurrence rate of only $4 \%$ in patients with one to three ENEs. However, this result was obtained based on the analysis of only a small number of patients $(n=23)$ with one recurrence. Furthermore, the patients enrolled in that study had an extraordinary high number of ENEs (median 8; IQR 1-57), and ENE was not further defined (9). Considering the prognostic significance of ENE, a detailed pathological examination to determine the presence of ENE should be performed. If ENE is detected, even if individuals have five or fewer positive LNs, patients should be considered to be in the intermediate-risk group. These patients may benefit from further treatment such as RAI treatment or additional TSH suppression.

Interestingly, the impact of ENE on recurrence differed according to the number of positive LNs, and disappeared in patients with more than five positive LNs. Similar to the results of the present study, ENE number in cancers of the oral cavity had a different impact according to the number of positive LNs. In a newly proposed nodal staging system based on recursive partitioning analysis, ENE was retained as a factor only in the case of a single positive LN, and had no impact on survival in patients with carcinomas of the oral cavity with more than two positive LNs (28). Although the exact reason why ENE affects recurrence only in patients with a few positive LNs is unclear, it may be that ENE promotes the spread of nodal disease at an early stage of N1 disease. Also, given that patients with intermediate risk usually receive RAI treatment, the assumption that PTC N1 patients with ENE may show a better response to RAI treatment could be another explanation. This notion is supported by a recent study on pancreatic cancer that demonstrated a better prognosis of patients with ENE when treated with adjuvant chemoradiation therapy but not chemotherapy alone (29). In addition, all three patients with the largest LNs ( $\geq 3 \mathrm{~cm}$; high-risk group in the current system) in this study were in the subgroup with more than five LNs and four or more ENEs. This suggests that the extremely high recurrence rate in patients with ENE in the previous study (recurrence rate of $32 \%$ in patients with $>3$ ENEs) (9) may have been influenced by LN size, which was not reported in that study.

Altogether, the presence of ENE appears to equate to an intermediate risk of recurrence within the current ATA system. After incorporating ENE into the current riskstratification system to create an alternative system, this system showed a better predictive ability than the current ATA risk-stratification system for predicting structural persistent/recurrent disease. The present results are useful when determining the initial treatment after surgery. However, the current study has several limitations that need to be taken into consideration. First, the results of this study might not apply to the long-term prognosis because of the relatively short follow-up period. However, early recurrence can provide enough information to determine the extent of initial treatment. Second, there were too few patients with five or fewer LNs and four or more ENEs to analyze whether ENE had a further impact on recurrence. Third, the possibility that structural persistent/recurrent disease could result from incomplete LN dissection cannot be excluded, although experienced surgeons carried out the operations according to established guidelines (30). The retrospective study design and the single-center nature of the study are additional limitations. Further research is therefore needed to confirm the findings. In addition, although the degree of individual ENE was not evaluated, evaluating a possible association between the degree of ENE and recurrence risk also seems to be a relevant topic for future studies.

In conclusion, the presence of ENE translates into an intermediate risk of recurrence in the current risk-stratification system. By incorporating ENE into the current risk-stratification system, physicians can make more accurate decisions about how to manage PTC N1 patients.

\section{Author Disclosure Statement}

The authors have no conflicts of interest to disclose.

\section{Supplementary Material}

Supplementary Figure S1

\section{References}

1. Haugen BR, Alexander EK, Bible KC, Doherty GM, Mandel SJ, Nikiforov YE, Pacini F, Randolph GW, Sawka AM, Schlumberger M, Schuff KG, Sherman SI, Sosa JA, Steward DL, Tuttle RM, Wartofsky L 20162015 American 
Thyroid Association management guidelines for adult patients with thyroid nodules and differentiated thyroid cancer: the American Thyroid Association Guidelines Task Force on Thyroid Nodules and Differentiated Thyroid Cancer. Thyroid 26:1-133.

2. Luchini C, Wood LD, Cheng L, Nottegar A, Stubbs B, Solmi M, Capelli P, Pea A, Sergi G, Manzato E, Fassan M, Bagante F, Bollschweiler E, Giacopuzzi S, Kaneko T, de Manzoni G, Barbareschi M, Scarpa A, Veronese N 2016 Extranodal extension of lymph node metastasis is a marker of poor prognosis in oesophageal cancer: a systematic review with meta-analysis. J Clin Pathol 2016 Jul 7 [Epub ahead of print]; DOI: 10.1136/jclinpath-2016203830.

3. Veronese N, Fassan M, Wood LD, Stubbs B, Solmi M, Capelli P, Pea A, Nottegar A, Sergi G, Manzato E, Carraro S, Maruzzo M, Cataldo I, Bagante F, Barbareschi M, Cheng L, Bencivenga M, de Manzoni G, Luchini C 2016 Extranodal extension of nodal metastases is a poor prognostic indicator in gastric cancer: a systematic review and metaanalysis. J Gastrointest Surg 20:1692-1698.

4. Veronese N, Nottegar A, Pea A, Solmi M, Stubbs B, Capelli P, Sergi G, Manzato E, Fassan M, Wood LD, Scarpa A, Luchini C 2016 Prognostic impact and implications of extracapsular lymph node involvement in colorectal cancer: a systematic review with meta-analysis. Ann Oncol 27:4248.

5. Roh JL, Park JW, Jeong J, Gong G, Cho KJ, Choi SH, Nam SY, Kim SY 2017 Extranodal extension of lymph node metastasis as a prognostic indicator of recurrence and survival in papillary thyroid carcinoma. J Surg Oncol 116: 450-458.

6. Lango M, Flieder D, Arrangoiz R, Veloski C, Yu JQ, Li T, Burtness B, Mehra R, Galloway T, Ridge JA 2013 Extranodal extension of metastatic papillary thyroid carcinoma: correlation with biochemical endpoints, nodal persistence, and systemic disease progression. Thyroid 23:1099-1105.

7. Nam SH, Roh JL, Gong G, Cho KJ, Choi SH, Nam SY, Kim SY 2018 Nodal factors predictive of recurrence after thyroidectomy and neck dissection for papillary thyroid carcinoma. Thyroid 28:88-95.

8. Veronese N, Luchini C, Nottegar A, Kaneko T, Sergi G, Manzato E, Solmi M, Scarpa A 2015 Prognostic impact of extra-nodal extension in thyroid cancer: a meta-analysis. J Surg Oncol 112:828-833.

9. Leboulleux S, Rubino C, Baudin E, Caillou B, Hartl DM, Bidart JM, Travagli JP, Schlumberger M 2005 Prognostic factors for persistent or recurrent disease of papillary thyroid carcinoma with neck lymph node metastases and/or tumor extension beyond the thyroid capsule at initial diagnosis. J Clin Endocrinol Metab 90:5723-5729.

10. Urken ML, Haser GC, Likhterov I, Wenig BM 2016 The impact of metastatic lymph nodes on risk stratification in differentiated thyroid cancer: have we reached a higher level of understanding? Thyroid 26:481-488.

11. Carty SE, Cooper DS, Doherty GM, Duh QY, Kloos RT, Mandel SJ, Randolph GW, Stack BC Jr, Steward DL, Terris DJ, Thompson GB, Tufano RP, Tuttle RM, Udelsman R 2009 Consensus statement on the terminology and classification of central neck dissection for thyroid cancer. Thyroid 19:1153-1158.

12. Caron NR, Clark OH 2005 Papillary thyroid cancer: surgical management of lymph node metastases. Curr Treat Options Oncol 6:311-322.
13. Jeon MJ, Kim WG, Jang EK, Choi YM, Song DE, Sung TY, Yoon JH, Chung KW, Hong SJ, Ryu JS, Han JM, Kim TY, Shong YK, Kim WB 2015 Sub-classification of lateral cervical lymph node metastasis in papillary thyroid carcinoma by pathologic criteria. PLoS One 10: e0133625.

14. Cabanillas ME, McFadden DG, Durante C 2016 Thyroid cancer. Lancet 388:2783-2795.

15. Kim TY, Kim WB, Kim ES, Ryu JS, Yeo JS, Kim SC, Hong SJ, Shong YK 2005 Serum thyroglobulin levels at the time of 131I remnant ablation just after thyroidectomy are useful for early prediction of clinical recurrence in low-risk patients with differentiated thyroid carcinoma. J Clin Endocrinol Metab 90:1440-1445.

16. Kim HI, Kim TH, Choe JH, Kim JH, Kim JS, Oh YL, Hahn SY, Shin JH, Jang HW, Kim YN, Kim H, Ahn HS, Kim K, Kim SW, Chung JH 2017 Restratification of survival prognosis of N1b papillary thyroid cancer by lateral lymph node ratio and largest lymph node size. Cancer Med 6: 2244-2251.

17. Oh HS, Ahn J, Song E, Han JM, Kim WG, Kim TY, Kim WB, Shong Y, Jeon M 2019 Individualized follow-up strategy for patients with indeterminate response to initial therapy for papillary thyroid carcinoma. Thyroid 29:209_ 215.

18. Harrell FE Jr, Lee KL, Mark DB 1996 Multivariable prognostic models: issues in developing models, evaluating assumptions and adequacy, and measuring and reducing errors. Stat Med 15:361-387.

19. Mangano DT, Tudor IC, Dietzel C 2006 The risk associated with aprotinin in cardiac surgery. N Engl J Med 354:353365.

20. Voss MH, Reising A, Cheng Y, Patel P, Marker M, Kuo F, Chan TA, Choueiri TK, Hsieh JJ, Hakimi AA, Motzer RJ 2018 Genomically annotated risk model for advanced renal-cell carcinoma: a retrospective cohort study. Lancet Oncol 19:1688-1698.

21. Kim H, Kim TH, Choe JH, Kim JH, Kim JS, Oh YL, Hahn SY, Shin JH, Chi SA, Jung SH, Kim YN, Kim HI, Kim SW, Chung JH 2017 Patterns of initial recurrence in completely resected papillary thyroid carcinoma. Thyroid 27:908-914.

22. Yamashita H, Noguchi S, Murakami N, Toda M, Uchino S, Watanabe S, Kawamoto H 1999 Extracapsular invasion of lymph node metastasis. A good indicator of disease recurrence and poor prognosis in patients with thyroid microcarcinoma. Cancer 86:842-849.

23. Randolph GW, Duh QY, Heller KS, LiVolsi VA, Mandel SJ, Steward DL, Tufano RP, Tuttle RM 2012 The prognostic significance of nodal metastases from papillary thyroid carcinoma can be stratified based on the size and number of metastatic lymph nodes, as well as the presence of extranodal extension. Thyroid 22:1144-1152.

24. Alpert EH, Wenig BM, Dewey EH, Su HK, Dos Reis L, Urken ML 2015 Size distribution of metastatic lymph nodes with extranodal extension in patients with papillary thyroid cancer: a pilot study. Thyroid 25:238-241.

25. Lee YM, Sung TY, Kim WB, Chung KW, Yoon JH, Hong SJ 2016 Risk factors for recurrence in patients with papillary thyroid carcinoma undergoing modified radical neck dissection. Br J Surg 103:1020-1025.

26. Wang LY, Palmer FL, Nixon IJ, Thomas D, Shah JP, Patel SG, Tuttle RM, Shaha AR, Ganly I 2014 Central lymph node characteristics predictive of outcome in pa- 
tients with differentiated thyroid cancer. Thyroid 24: 1790-1795.

27. Lee CW, Roh JL, Gong G, Cho KJ, Choi SH, Nam SY, Kim SY 2015 Risk factors for recurrence of papillary thyroid carcinoma with clinically node-positive lateral neck. Ann Surg Oncol 22:117-124.

28. Ho AS, Kim S, Tighiouart M, Gudino C, Mita A, Scher KS, Laury A, Prasad R, Shiao SL, Van Eyk JE, Zumsteg ZS 2017 Metastatic lymph node burden and survival in oral cavity cancer. J Clin Oncol 35:3601-3609.

29. Sergeant G, Ectors N, Fieuws S, Aerts R, Topal B 2009 Prognostic relevance of extracapsular lymph node involvement in pancreatic ductal adenocarcinoma. Ann Surg Oncol 16:3070-3079.

30. Robinson TJ, Thomas S, Dinan MA, Roman S, Sosa JA, Hyslop T 2016 How many lymph nodes are enough? Assessing the adequacy of lymph node yield for papillary thyroid cancer. J Clin Oncol 34:3434-3439.
Address correspondence to:

Tae Hyuk Kim, MD, PhD

Division of Endocrinology and Metabolism

Department of Medicine

Thyroid Center, Samsung Medical Center Sungkyunkwan University School of Medicine

Seoul 06351

Republic of Korea

E-mail: taehyukmd.kim@samsung.com

Young Lyun Oh, MD, PhD

Department of Pathology

Samsung Medical Center

Sungkyunkwan University School of Medicine

Seoul 06351

Republic of Korea

E-mail: yl.oh@samsung.com 